\title{
Blazars: Clues to jet physics
}

\author{
Rita M. Sambruna \\ Pennsylvania State University, 525 Davey Lab, University Park, PA \\ 16802
}

\begin{abstract}
.
Being dominated by non-thermal emission from aligned relativistic jets, blazars allow us to elucidate the physics of extragalactic jets, and, ultimately, how energy is extracted from the central black hole. Crucial information about jet structure is provided by the spectral energy distributions from radio to $\gamma$-rays, their trends with luminosity, and correlated multifrequency variability. Since blazar jets have broad implications for all radio-loud (and possibly radio-quiet) AGNs, we also need to understand their circumnuclear structure, especially the details of the physical and dynamical conditions of the highly ionized gas on sub-pc scales, which could be directly related to jet formation and radiative power. Eventually, the bulk of information provided by blazars will help us clarify the origin of the radio-loud/radio-quiet AGN dichotomy, one of the most outstanding open issues of extragalactic astrophysics.
\end{abstract}

\section{The importance of blazars for all AGNs}

Blazars (including BL Lacertae objects and quasar-like blazars) are among the most violent manifestations of activity in galaxies. Their defining properties include large luminosities emitted on short timescales, high and variable polarization degrees in optical and IR, smooth continuum emission from radio to $\gamma$-ray energies. These properties are best explained as non-thermal emission from a relativistic jet oriented close to the line of sight (Blandford \& Rees 1978). Compelling evidence for relativistic beaming of the emitted radiation is provided by the strong and rapidly variable $\gamma$-ray emission observed in many blazars in recent years (Hartman 1998).

Today, the relevance of blazars for all AGNs is increasingly recognized as it becomes evident that relativistic jets are a feature of accretion in all radio-loud AGNs (Urry \& Padovani 1995), and possibly also in their radio-quiet counterparts (e.g., Falcke et al. 1996), while superluminal ejecta are also present on Galactic scales (Mirabel \& Rodriguez 1998). What originally made blazars appear exotic and of little appeal to the astronomical community, now turns out to be their major strength. We need to understand how jets are formed and work if we want to understand how energy is extracted from the central black hole in AGNs (Krolik, this volume). Blazars provide us with a direct probe of this fundamental issue. 

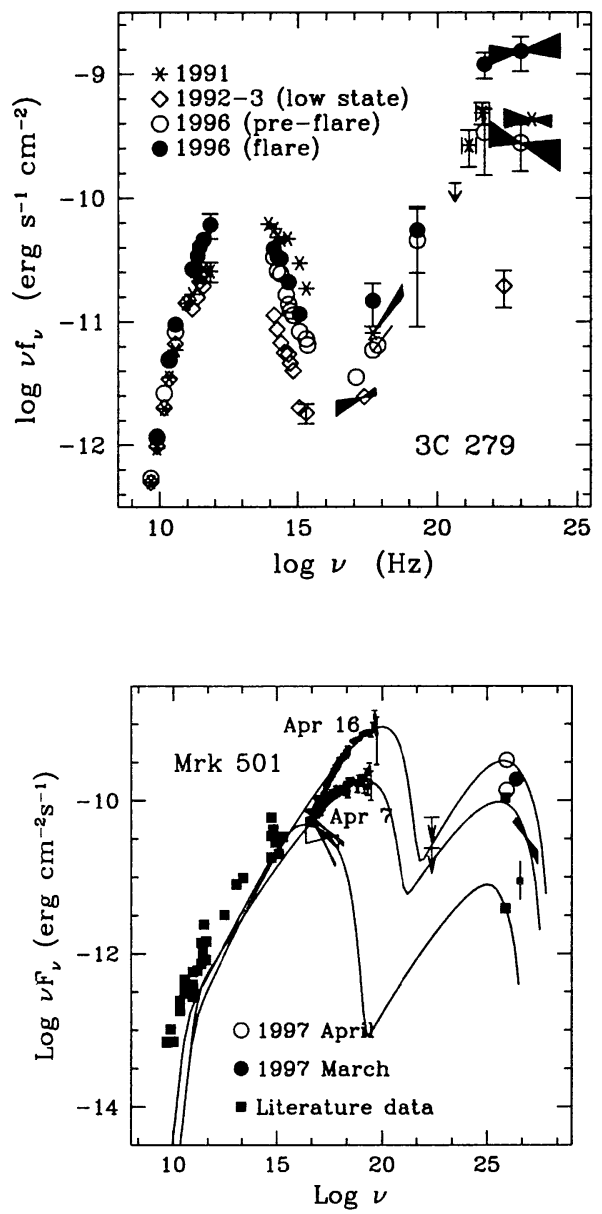

Figure 1. Spectral energy distributions and variability of the red blazar 3C279 (top) and blue blazar Mrk 501 (bottom). The continua are characterized by two broad humps peaking at different wavelengths in the two cases, respectively. Variability is more pronounced above the peaks at both low and high energies (data from Wehrle et al. 1998 and Pian et al. 1998). 


\section{Spectral energy distributions: The blazar family}

First clues about jet physics are provided by blazars' spectral energy distributions (SEDs). These typically exhibit two broad humps (Fig. 1): the first one peaks at IR/optical in "red" blazars and at UV/X-rays in their "blue" counterparts (Giommi et al. 1994), and is due to synchrotron emission (Sambruna et al. 1996). The second component extends from X-rays up to $\mathrm{GeV} / \mathrm{TeV}$ energies, and its origin is less well understood. One promising explanation is inverse Compton (IC) scattering of ambient photons, either internal (synchrotron-self Compton, SSC; Maraschi et al. 1992) or external to the jet (External Compton, EC; Dermer \& Schlickeiser 1993; Sikora et al. 1994; Ghisellini \& Madau 1996).

Red and blue jets are just the extrema of a continuous population. Deep multicolor surveys are finding blazars with intermediate spectral shapes (LaurentMeuhleisen et al. 1998; Perlman et al. 1998). Spectral trends with bolometric luminosity are observed in existing samples: from red to blue blazars the luminosity decreases and the synchrotron and IC peak frequencies decrease proportionately. Red sources also appear to have a larger $\gamma$-ray dominance and more luminous broad emission lines (Sambruna et al. 1996; Sambruna 1997; Fossati et al. 1998).

In the context of a simple homogeneous model, where a single electron population is responsible for producing both components through synchrotron and IC processes, the spectral trends can be interpreted as a change of a few jet physical parameters: the higher synchrotron peak frequencies of blue blazars call for higher magnetic fields or/and electron energies than red sources (Sambruna et al. 1996). It is also possible that red blazars have larger luminosities and lower electron energies as a result of a more efficient cooling through EC scattering (Ghisellini et al. 1998). In this case, the paradigm would be that the type of electron cooling depends on the density of the external photons, with the SED continuity hinting to a large spread of physical properties of the jet ambient medium, commensurate with the observed line properties (Scarpa \& Falomo 1997).

However, the luminosity trends are most likely affected by strong biases, especially at $\gamma$-rays, where the limited sensitivity of current $\mathrm{GeV}$ detectors combined with large variability could exaggerate the $\gamma$-ray dominance in the most distant red blazars (and bias toward EC-dominated sources, where the $\gamma$-rays are more beamed). In blue blazars Klein-Nishina cutoffs kick in at $\mathrm{TeV}$ energies and the ratio of the synchrotron to IC peak is only approximately constant. Finally, recent deep surveys show the existence of an unexpected population of blue blazars with strong emission lines (Perlman et al. 1998; see also Sambruna 1997). While the new spectral trends will be tested more extensively in the future, a clear message emerges from the current data: blazars exhibit a rich variety of spectral behaviors, which point to a diversity of jet physical conditions rather than being due to beaming/orientation effects only (Sambruna et al. 1996; Georganopoulos \& Marscher 1998; Kubo et al. 1998). 


\section{Correlated multiwavelength variability: Looking inside the jet}

One crucial still unanswered question is the origin of the $\mathrm{GeV}$ and $\mathrm{TeV}$ emission. An alternative to the SSC and EC scenarios $(\S 2)$ is provided by the proton-induced cascades models (PIC; Protheroe \& Biermann 1997; Mannheim $\&$ Biermann 1992), where the $\gamma$-rays are direct synchrotron emission from ultrarelativistic electrons while the $\mathrm{X}$-rays are emitted by less energetic electrons originating from pair cascades initiated by protons. The viability of hadronic models, at least in red blazars, may be supported by recent observations of circular radio polarization (Wardle et al. 1998). Clearly, distinguishing between the IC and PIC scenarios is of central importance to understand the jet composition and how energy is transported away from the central black hole.

Correlated multifrequency variability provides a way to this end, since the different models make distinct predictions for the relative flare amplitudes and for the time lags. In a one-zone homogeneous approximation, the synchrotron plus IC models predict: 1) strongly correlated variability of the fluxes at the low- and high-energy peaks with no time lags, since the same electron population is responsible for emitting both spectral components; 2) simple and yet precise relationships for the relative amplitudes of the synchrotron and IC flares depending (for a fixed beaming factor $\delta$ ) on the change of electrons and/or seed photons (Ghisellini \& Maraschi 1996); 3) accurate shapes of the synchrotron and IC flares depending on a few source typical timescales (Chiaberge \& Ghisellini 1998) and parameters (Romanova \& Lovelace 1997; Böttcher \& Dermer 1998); 4) lags between soft and hard X-rays, reflecting the electron radiative time $\left(\mathrm{t}_{r} \propto E^{-1 / 2} B^{-3 / 2} \delta^{-1 / 2}\right)$, from which the local magnetic field $B$ can be derived (modulo $\delta$; Takahashi et al. 1996); 5) defined time-dependent spectra, according to how fast electrons are accelerated compared to $t_{r}$ (Kirk et al. 1998).

In contrast, PIC models allow more freedom: lags of either sign between long and short wavelengths are possible, as well as more arbitrary relations in the flare relative amplitudes, because the relative energy deposited in electrons and protons is a free parameter, and so the details of the ensuing pair production are less constrained.

The target selection for multifrequency campaigns is strongly constrained by the $\gamma$-ray band, where only a handful red blazars are bright enough to be monitored at $\mathrm{GeV}$ energies. At $\mathrm{TeV}$ even fewer sources are detected, while $\mathrm{TeV}$ photons from distant blazars may be difficult to observe because of their interaction with the IR background (Salomon \& Stecker 1994). Despite the intrinsic difficulty of such campaigns, now severely undermined by the death of the EGRET CGRO detector, a few blazars were monitored well enough to allow comparison of models to the data.

Results for red blazars. The multi-epoch SEDs of 3C279, one of the most luminous $\mathrm{GeV}$ sources in the sky (Fig. 1), shows indeed correlated variability at IR/optical and $\mathrm{GeV}$, supporting IC models. The amplitude of the $\mathrm{GeV}$ variation goes quadratically with the optical flux in early campaigns, indicating a variation of the electron density as the cause of the flare in the SSC model (Maraschi et al. 1994) or a change of beaming factor in the EC model. During the 1996 campaign, however, the $\gamma$-rays varied more than quadratically than the optical/IR flux, and exactly (within 1 day) simultaneously to the X-rays (Wehrle et al. 1998), with 
the rapid decay time of the GeV flare favoring EC scenarios (Ghisellini \& Madau 1996).

A new red candidate for multifrequency campaigns is BL Lac itself, which underwent a strong $\mathrm{GeV}$ and optical outburst in $1997 \mathrm{July}$ (Bloom et al. 1997). The $\gamma$-ray light curve shows a strong flare possibly anticipating the optical flare by $\sim 0.5$ days; however, the poor $\gamma$-ray sampling prevents any firm conclusion. Interestingly, a broad $\mathrm{H} \alpha$ emission line was detected in BL Lac (Vermeulen et al. 1995), which increased in luminosity at the time of the optical/GeV outburst, suggesting that EC models are responsible for producing the flux above a few $\mathrm{MeV}$. This is indeed supported by a detailed modeling of the SED (Sambruna et al. 1999).

Results for blue blazars. Mrk 501, one of only four extragalactic TeV sources (all blazars), flared dramatically at $\mathrm{TeV}$ and X-rays in 1997 April (Aharonian et al. 1997; Catanese et al. 1997); TeV and X-ray light curves track each other well, with no lags larger than 1 day, confirming that the $\mathrm{TeV}$ photons are produced by the same electrons responsible for the X-rays. A similar behavior was observed also during our recently concluded campaign in 1998 June. As shown in Fig. 1, during the $1997 \mathrm{TeV}$ flare the synchrotron peak shifted forward by 2 orders of magnitude in correspondence to a dramatic flattening of the X-ray continuum, implying large acceleration events (Pian et al. 1998). Mrk 501 is an eloquent example of the dramatic powers that can be reached in blazars.

An ideal candidate for studying the energy-dependence of the synchrotron flares is PKS 2155-304, one of the brightest and most rapidly variable BL Lacs at optical through X-ray wavelengths, recently detected in $\mathrm{TeV}$ (Chadwick et al. 1998). Previous campaigns (Edelson et al. 1995; Urry et al. 1997) detected correlated variability at optical through X-ray wavelengths, and established that the shorter wavelengths generally vary first, with lags of the order of a few hours to a few days. The measured 1 hour delay between soft and hard X-rays in 1994 May yields $B \sim 0.1$ Gauss (Urry et al. 1997), similar to Mrk 421 (Takahashi et al. 1996).

The multifrequency experiment for PKS 2155-304 was repeated in 1996 May (Fig. 2), with better sampling in X-rays but worse at longer wavelengths. Complex variability was observed, with several short flares superposed to a longer trend. The flares become more symmetric at decreasing wavelengths, roughly consistent with a homogeneous synchrotron scenario where the radiative time at high energies is fast, and the light travel time limits both the rise and decay times (Chiaberge \& Ghisellini 1998). No lags larger than $\sim 1.5$ hours were observed. The X-ray hardness ratios (Fig. 2) follow a general clock-wise loop with intensity, indicating that acceleration is fast and the spectrum is controlled by radiative cooling, with superposed events of anti-clockwise cycles, when cooling and acceleration are in equilibrium (Kirk et al. 1998).

What did we learn? Current multifrequency data support models which predict correlated variability at the low- and high-energy peaks in both blue and red blazars. While it proved difficult to distinguish among models based on the relative flare amplitudes, due to the large number of free parameters involved, detection of time lags and flare shape promises to be more efficient to this end, but is limited at present by sampling constraints, especially at $\gamma$-rays. 

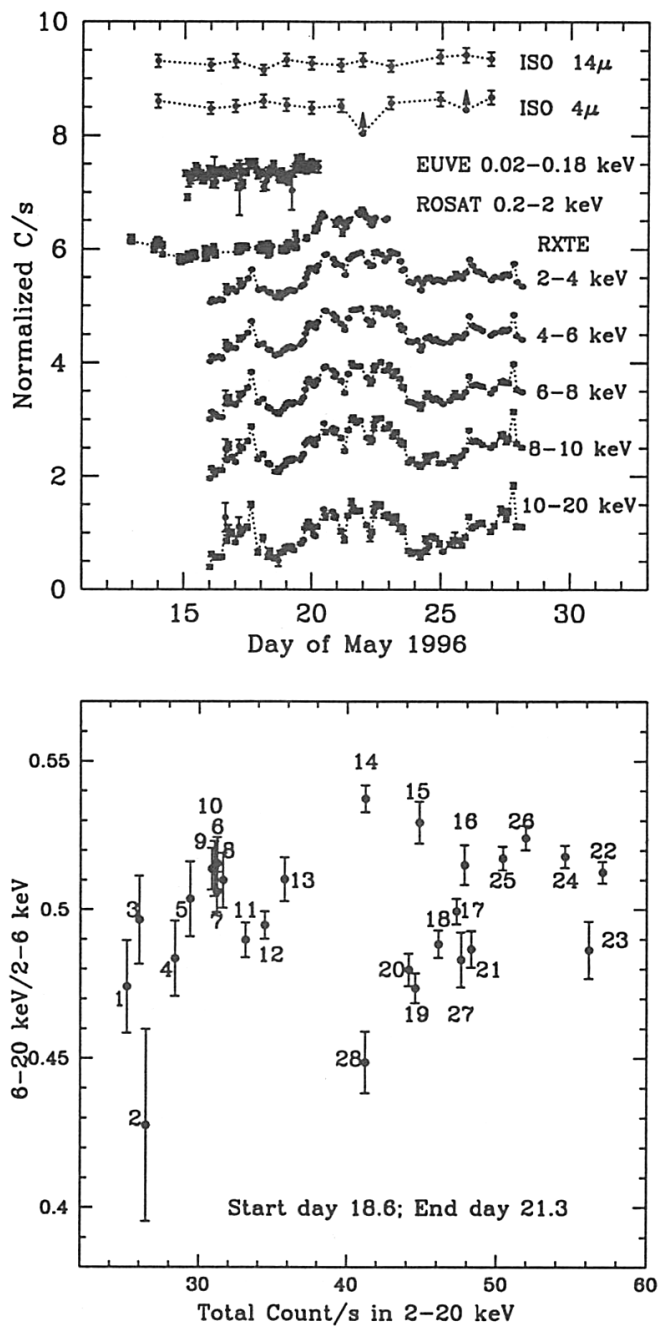

Figure 2. Multifrequency variability of PKS 2155-304 in 1996 May. Top: IR to X-ray light curves, normalized to their mean fluxes and arbitrarily offset (data from papers in prep. by Bertone, Brinkmann, Marshall, Sambruna). No lags are detected larger than the RXTE binning time $(90 \mathrm{~min})$; the flares have larger amplitudes and more symmetric shapes at increasing energies. Bottom: An example of X-ray hardness ratios vs. intensity, numbered chronologically. The spectral changes follow a general clock-wise cycle, with anti-clock-wise smaller loops. This is a diagnostic of the acceleration processes in the emission region. 
Spectral variability at X-rays is a powerful diagnostic of the acceleration events in the jets, and can be studied well with RXTE and SAX.

\section{Blazar environment: Where jets form}

The study of blazar environment has recently received much attention, as the importance of the external medium for jet formation and deceleration (De Young 1993; Bicknell 1995) and for jet radiative power (Dermer \& Chiang 1998) is increasingly recognized. IR and optical studies from the ground (see Heidt 1998 for a review) and with HST (Urry et al. 1999 and references therein) show that BL Lacs generally lie in poor clusters (Abell richness 0-1), and that their host galaxies are smooth, luminous ellipticals, with no differences between red and blue sources. The host galaxies of BL Lacs are very similar to those of their putative parent populations, Fanaroff-Riley I galaxies, of matching extended radio powers (Urry et al. 1999b, in prep.), supporting unified models for radioloud AGNs. Substantial progress in the study of blazar environs will be achieved in the near future with $\mathrm{AXAF}^{1}$ observations in X-rays, which will allow us to measure directly the medium properties (temperature, density, abundances) and probe in depth the gravitational potential.

High-energy absorption in BL Lacs. A powerful probe of the inner (pc/subpc scales) jet environs is provided by high-resolution observations of a few bright BL Lacs at X-ray and EUV wavelengths. Broad absorption features were detected in PKS 2155-304 and Mrk 421, the only two blazars observed at grating resolutions in soft X-rays (Canizares \& Kruper 1984) and EUV (Königl et al. 1995; Kartje et al. 1997), suggesting absorption in highly ionized gas outflowing with mildly relativistic velocities $(v \lesssim 0.1 c)$. Ionized absorption was also detected in BBXRT and ASCA observations of a few more BL Lacs (Sambruna et al. 1997; Sambruna \& Mushotzky 1998), confirming earlier claims (Madejski et al. 1992).

The origin of the absorbing medium is still a speculation. One possibility is the disk-driven hydromagnetic wind model (Königl \& Kartje 1994), based on the mechanism originally proposed by Blandford \& Payne (1982) to explain the origin of relativistic jets (Lovelace, this volume): clouds of gas are lifted from the accretion disk surface by the magnetic field lines until they intercept the beamed jet radiation, giving rise to absorption when crossing the line of sight. Alternatively, absorption could originate in matter swept up by the jet outer edge (Krolik et al. 1985), perhaps the same decelerating medium required by unification schemes (Bicknell 1995). At the current low spectral resolution and sensitivity there is no way to discriminate among the possible models; however, this will be trivial with the gratings onboard the future AXAF and X-ray MultiMirror missions, which will allow us to measure precisely the absorber properties and trace a detailed portrait of the inner jet environs.

\footnotetext{
${ }^{1}$ The Advanced X-ray Astrophysical Facility will carry, among its various detectors, a set of CCD imagers with spectroscopic capabilities, with a much narrower PSF (FWHM $\lesssim 1$ arcsec) and lower intrinsic background than ROSAT.
} 


\section{Future work}

While recent years have seen gigantic progress in our understanding of the blazar phenomenon, much more work remains to be done.

We need to test more extensively the luminosity/distance spectral trends, in particular at $\gamma$-rays, with larger complete samples, fully addressing the various selection effects. More TeV observations, where we currently have only 4 detected sources, are badly needed, as well as more precise determinations of the $\mathrm{TeV}$ spectra in order to quantify better the position of the IC peak in blue blazars.

We need to intensify our efforts in multiwavelength monitoring of blue and (more) red blazars, in particular to measure more precisely the time lags down to the smallest accessible scales, and the flare shapes both at low and high energies. With the death of EGRET on CGRO, the short-term future of multifrequency campaigns relies only on $\mathrm{TeV}$ blazars.

Blazar environment studies at IR, optical, and X-ray wavelengths with current and future high-resolution satellites (HST, NGST, AXAF, XMM) will address the AGN/host galaxy connection, in particular the role of the ambient medium for jet formation and collimation, and for fueling the central black hole.

With the advent of new technical resources of improved sensitivity and resolution in a large wavelength range, the future holds great promise for blazars. Eventually, the bulk of information provided by blazars will help us shed light on the origin of jets and, ultimately, of the radio-loud/radio-quiet AGN dichotomy, one of the most outstanding open problems of extragalactic astrophysics.

Acknowledgments. This work was supported by NASA grants NAS38252, NAG-3313, NAG5-7121, and by an IAU travel grant. I thank Meg Urry for interesting discussions, and all my colleagues at the conference for a memorable meeting.

\section{References}

Aharonian, F. et al. 1997, A\&A, 327, L5

Bicknell, G. V., 1995, ApJS, 101, 29

Blandford, R.D. \& Payne, D.G. 1982, MNRAS, 199, 883

Blandford, R.D. \& Rees, M.J. 1978, in Pittsburgh Conference on BL Lac Objects, A.M.Wolfe, Univ. Pittsburgh Press, 1978, p.328

Bloom, S. D. et al. 1997, ApJ, 490, L145

Böttcher, M. \& Dermer, C.D. 1998, ApJ, 501, L51

Canizares, C.R. \& Kruper, J. 1984, ApJ, 278, L99

Catanese, M. et al. 1997, ApJ, 487, L143

Chadwick, P.M. et al. 1998, ApJ, in press, astro-ph/9810209

Chiaberge, M. \& Ghisellini, G. 1998, MNRAS, subm., astro-ph/9810263

De Young, D. S. 1993, ApJ, 405, L13

Dermer, C.D. \& Chiang, J. 1998, astro-ph/9810222

Dermer, C.D. \& Schlickeiser, R. 1993, ApJ, 416, 458 
Edelson, R. et al. 1995, ApJ, 438, 120

Falcke, H. et al. 1996, ApJ, 473, L13

Fossati, G. et al. 1998, MNRAS, 299, 433

Georganopoulos, M. \& Marscher, A. 1998, ApJ, 506, 621

Ghisellini, G. et al. 1998, MNRAS, in press, astro-ph/9807317

Ghisellini, G. \& Madau, P. 1996, MNRAS, 280, 67

Ghisellini, G. \& Maraschi, M. 1996, in Blazar Continuum Variability, H.R.Miller, J.R.Webb, \& J.C.Noble, ASP Conf. Series, Vol. 110, 436

Giommi, P., Ansari, S.G., \& Micol, A. 1995, A\&ASupp., 109, 267

Hartman, R.C. 1998, in BL Lac Phenomenon, Eds. A.Sillanpaa \& L.O.Takalo, PASP Conf. Series, in press

Heidt, J. 1998, in BL Lac Phenomenon, Eds. A.Sillanpaa \& L.O.Takalo, PASP Conf. Series, in press

Kartje, J.F. et al. 1997, ApJ, 474, 630

Kirk, J.G., Rieger, F.M., \& Mastichiadis, A. 1998, A\&A, 333, 452

Königl, A. et al. 1995, ApJ, 446, 598

Königl, A. \& Kartje, J.F. 1994, ApJ, 434, 446

Krolik, J.H. et al. 1985, ApJ, 295, 104

Kubo, H. et al. 1998, ApJ, 504, 693

Laurent-Muehleisen, S.A. et al. 1998, ApJS, 118, 127

Madejski, G. et al. 1991, ApJ, 370, 198

Mannheim, K. \& Biermann, P.L. 1992, A\&A, 253, L21

Maraschi, L. et al. 1994, ApJ, 435, L91

Maraschi, L., Ghisellini, G., \& Celotti, A. 1992, ApJ, 397, L5

Mirabel, I.F. \& Rodriguez, L.F. 1998, Nature, 392, 673

Perlman, E.S. et al. 1998, AJ, 115, 1253

Pian, E. et al. 1998, ApJ, 492, L17

Protheroe, R.J. \& Bierman, P.L. 1997, APh, 6, 293

Romanova, M.M. \& Lovelace, R.V.E. 1997, ApJ, 475, 97

Salomon, H.M. \& Stecker, F.W. 1994, ApJ, 430, L21

Sambruna, R.M. et al. 1999, ApJ, in press, astro-ph/9810319

Sambruna, R.M. \& Mushotzky, R.F. 1998, ApJ, 502, 630

Sambruna, R.M. 1997, ApJ, 474, 639

Sambruna, R.M. et al. 1997, ApJ, 483, 774

Sambruna, R.M., Maraschi, L., \& Urry, C.M. 1996, ApJ, 463, 444

Scarpa, R. \& Falomo, R. 1997, A\&A, 325, 109

Sikora, M., Begelman, M.C., \& Rees, M.J. 1994, ApJ, 421, 153

Takahashi, T. et al. 1996, ApJ, 470, L89

Urry, C.M. et al. 1999, ApJ, in press, astro-ph/9809030

Urry, C.M. et al. 1997, ApJ, 486, 799

Urry, C.M. \& Padovani, P. 1995, PASP, 107, 803 
Vermeulen, R.C. et al. 1995, ApJ, 452, L5

Wardle, J.F.C. et al. 1998, Nature, 395, 457

Wehrle, A. E. et al. 1998, ApJ, 497, 178

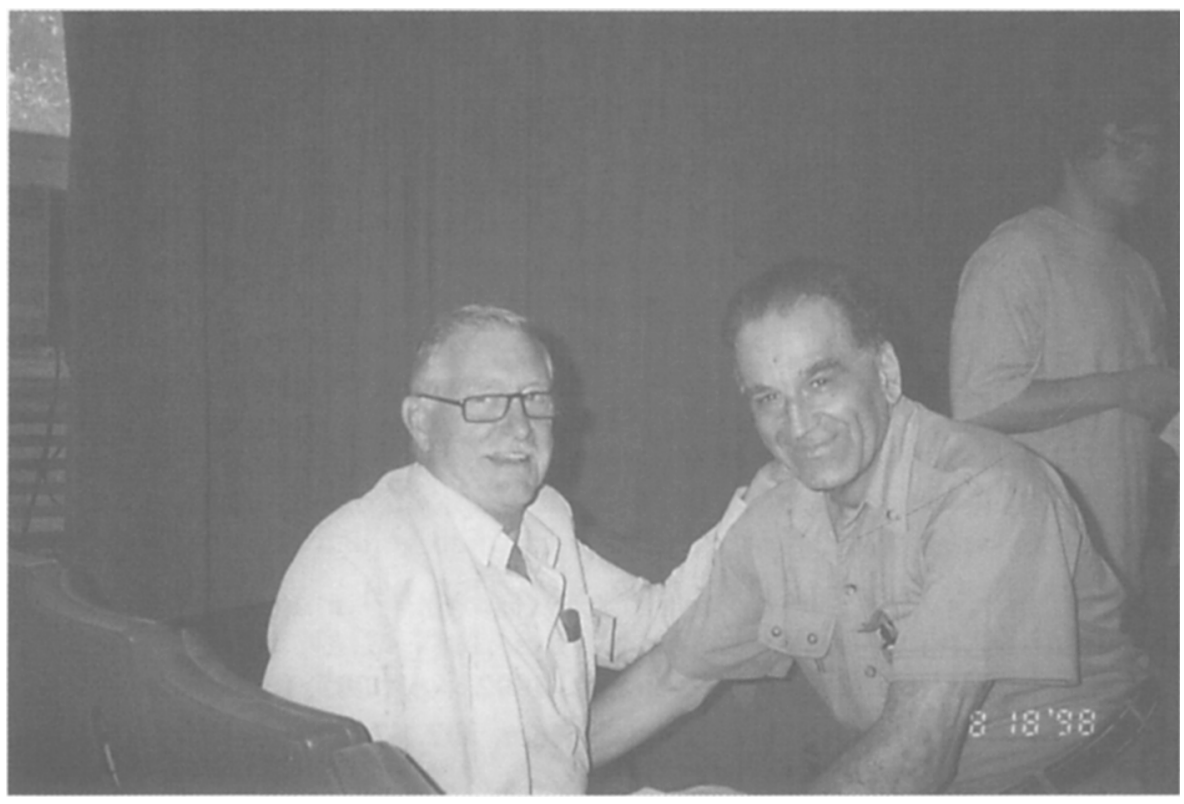

Halton Arp and Ed Khachikian 